\title{
Quality of data in the Manchester orthopaedic database
}

\author{
J L Barrie, D R Marsh
}

\section{Abstract}

Objective-To determine the completeness and accuracy of data in a computerised clinical information system (Manchester orthopaedic database) in comparison with the data available through the Hospital Activity Analysis.

Design-Retrospective review of case notes, computer data, and Hospital Activity Analysis data.

Setting-Orthopaedic unit in a district general hospital in Manchester.

Subjects -200 random patient records distributed through the period of use of the computer system (1 October 1988 to 31 March 1990) and 121 records for random admissions between 1 April 1989 and 31 March 1990,71 of which were included in the previous sample.

Main outcome measures-Conformity of the computer record key words and Hospital Activity Analysis codes to an ideal key word record and ideal code record drawn up by one investigator from the clinical notes; overall quality (completeness times accuracy).

Results-Overall completeness of the data in the orthopaedic database was $62 \%$ and the accuracy was $96 \%$. Completeness improved after feedback to doctors on the use of key words in regular audit meetings. Completeness was higher in inpatient than outpatient records $(69.9 \% v 53.7 \%, p<0.001)$ and when a new key word was required compared with missing and incorrect key words (both $\mathbf{p}<0.001$ ). Completeness was lower when the key word was required of a senior registrar $(p<0 \cdot 05)$. Accuracy was not significantly different. The completeness of Hospital Activity Analysis data was $\mathbf{9 0 . 5 \%}$ and accuracy $69 \cdot 5 \%$. Thus the overall data quality was similar in both systems.

Conclusions - Even in a system designed for simple and efficient data capture, compliance by users was poor. Accuracy was high, suggesting that users understood the principles of data entry. Completeness of data capture can be improved by providing feedback to users on use of the system and performance. Improvements in future versions of the software should improve performance.

University of Manchester, Manchester M13 9PT

J L Barrie, FRCSED, tutor in orthopaedic surgery

D R Marsh, MD, senior

lecturer in orthopaedic surgery

Correspondence to: $\mathrm{Mr} \mathrm{J} \mathrm{L}$ Barrie, University

Department of Orthopaedic Surgery, Clinical Sciences

Building, Hope Hospital

Eccles Old Road, Salford.

$B M \mathcal{F}$ 1992;304:159-62

\section{Introduction}

Many patient record systems have been described for clinical use ${ }^{1-3}$; most entail coding of patient information at the end of an inpatient stay by a specially employed person ${ }^{4}$ or by busy junior medical staff. ${ }^{2}$ It seems to be assumed by the users of most of these systems that the information contained is complete and accurate, although Brand et al noted that their orthopaedic database at Yale was "less than $100 \%$ complete," 3 and Lyons and Gumpert, using the program "Dunnfile," found $30 \%$ of data on hospital admissions to be missing." Kuslich and Peck, accounting for resistance to computerisation, criticised previous attempts at electronic data capture for "ignoring important human factors ... and longstanding information transfer mechanisms."

The Manchester orthopaedic database is a computer program which comprises both a word processor capable of generating a wide variety of clinical documents and a clinical database from which patient information can be extracted for research and audit. It answers Kuslich and Peck's criticisms by making use of traditional dictated orthopaedic notes for information capture. It also streamlines the medical secretary's job so that the small additional workload involved in entering "key words" (see below) to the database is more than compensated for by relief from numerous routine aspects of typing.

The details of the data structure and data capture process in the database have been described elsewhere. ${ }^{6}$ Briefly, data are captured in the form of key words, which are dictated as an addendum to routine clinical notes, operation notes, and so on. Key words are standard English terms used in a structured manner. They are familiar to all orthopaedic surgeons: there is no need for complex coding manuals. There are three main types of key word: diagnostic, operative, and complication. Two special types of key word (admission and procedure) will not be covered further in this paper. Dictionaries of key words and explanatory wallcharts are freely available wherever dictation is done. At each site where the database is in use a member of staff acts as "system coordinator," regularly checking the key word record, controlling the introduction of new key words and correcting any errors found in the existing key word record.

The database was introduced at Ancoats Hospital, Manchester, in October 1988. This was its first implementation in a general orthopaedic unit. We analysed our first 18 months' experience to assess the quality of the data collected.

\section{Materials and methods}

One author (JLB) reviewed, firstly, 200 randomly selected sets of case notes representing orthopaedic consultations (inpatient, outpatient, or both) between 1 October 1988 and 31 March 1990. The cases were selected by random generation of hospital registration numbers. The case folders were then retrieved from the medical records department at Ancoats Hospital or obtained from other hospitals in North Manchester District. In two cases the case folders proved impossible to trace, and other numbers were selected.

A "key word opportunity" was defined as any occasion when a key word could have been added to the database or changed for any reason. A single clinical encounter with a patient may generate more than one key word opportunity. For example, a new patient with osteoarthritis of the hip and knee who has had a previous knee replacement elsewhere would require the key words osteoarthritis-hip, osteoarthritis-knee, and arthroplasty-knee, each key word representing a 
separate key word opportunity, as each may be included or omitted separately.

For each clinical encounter the "ideal key word record" was derived from the clinical notes. This represented the most accurate set of key words possible on the evidence of the notes up to that point, in the reviewer's judgment. (It was derived without sight of the existing key word record.) The ideal key word record was compared with the actual record. Completeness was defined as the ratio of the number of key words present to the number which should have been present at that point. Accuracy was defined as the ratio of the number of key words present and correct to the total number of key words present. Overall data quality was the product of these two categories.

For each key word opportunity the type of key word (diagnostic, operative, admission, or complication), the indication for dictation (new, missing, or wrong), the site of use (inpatient or outpatient), the date of use, and the grade of user were noted.

At the end of treatment for each individual problem the actual key word record for that problem was again compared with its ideal key word record to give a final measure of completeness and accuracy for that episode, reflecting not only the input of key words but the effects of correction of errors and omissions.

The same author reviewed the notes for 121 random admissions to the orthopaedic unit at Ancoats Hospital between 1 April 1989 and 31 March 1990: 71 had been included in the previous sample, and the 50 others were generated by the same technique, only cases that included one or more admissions within the appropriate period being accepted.

The key word record at the end of each admission was compared with its ideal key word record. For comparison with the standard hospital coding techniques the coding record derived from the Hospital Activity Analysis card (1 April to 30 September 1989) and the Körner episodes report (1 October 1989 to 31 March 1990) were similarly compared with an ideal code record generated in the same way as for the ideal key word record. In each case the last opportunity for key word entry and the date of coding were noted.

Statistical analysis was by $\chi^{2}$ and Student $t$ tests.

\section{Results}

Table I summarises the findings of the case notes review. Key words required in an inpatient setting were more likely to be provided $(p<0.001)$, but there was no significant difference in accuracy between inpatient and outpatient key words.

TABLE I-Completeness and accuracy of key words in case notes of outpatients and inpatients

\begin{tabular}{lccc}
\hline & All patients & Inpatients & Outpatients \\
\hline No of key word opportunities & 605 & 296 & 309 \\
No of key words present & 373 & 207 & 166 \\
Completeness (\%) & $61 \cdot 7$ & 69.9 & 53.7 \\
No of key words correct & 360 & 198 & 162 \\
Accuracy (\%) & 96.5 & 95.6 & 97.6 \\
\hline
\end{tabular}

Difference in completeness, outpatients $v$ inpatients $p<0.001$; all other comparisons not significant.

In December 1989 we began to discuss key words both informally and formally at the monthly unit audit meeting, providing feedback on accuracy and discussing problems. Figure 1 shows how completeness changed with time, at the end of each key word opportunity and at the end of episodes of care, and how it responded to feedback. Table II shows completeness of key words related to different key word types; there was a particular failure to collect key words relating to complications.

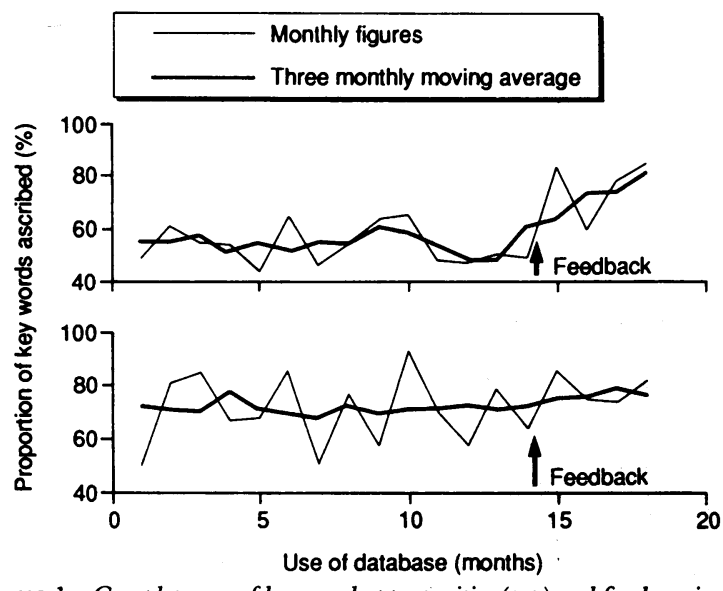

FIG 1-Completeness of key word opportunities (top) and final entries (bottom) by months of use of database

TABLE II-Completeness of key words by key word type

\begin{tabular}{lccc}
\hline & \multicolumn{3}{c}{ Key word type } \\
\cline { 2 - 4 } & Diagnostic & Operative & Complication \\
\hline No of key word opportunities & 433 & 111 & 61 \\
No of key words present & 254 & 91 & 28 \\
Completeness (\%) & 58.7 & 82.0 & 45.9 \\
\hline
\end{tabular}

Figure 2 shows how the accuracy of key words varied with time and table III the variation with key word type. Diagnostic accuracy did not vary in the same way as completeness, and the levels attained were much more satisfactory.

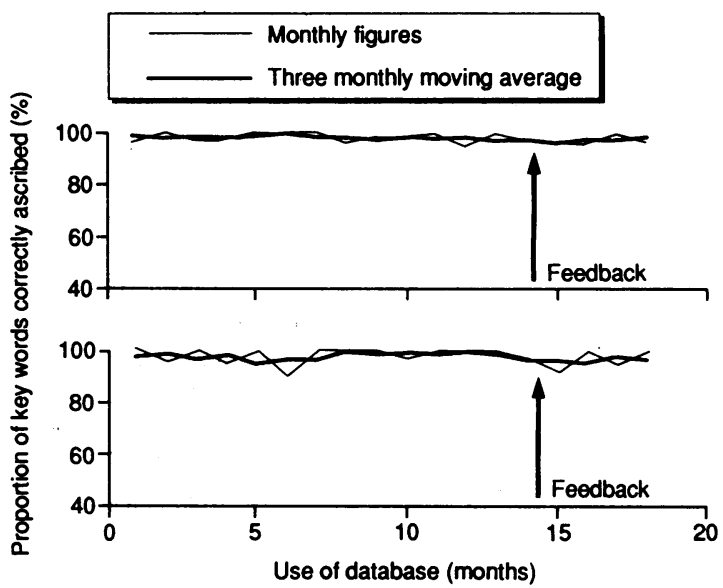

FIG 2-Accuracy of key word opportunities (top) and final entries (bottom) by months of use of database

TABLE III - Accuracy of key words by key word types

\begin{tabular}{lccc}
\hline & \multicolumn{3}{c}{ Key word type } \\
\cline { 2 - 4 } & Diagnostic & Operative & Complication \\
\hline No of key words present & 254 & 91 & 28 \\
No correct & 245 & 89 & 26 \\
Accuracy (\%) & 96.5 & $97 \cdot 8$ & 92.9 \\
\hline
\end{tabular}

When completeness and accuracy for different key word indications were compared (table IV) the results indicated that users were significantly better at dictating new key words than at inserting missing ones or correcting errors (both $\mathrm{p}<0.001$ ). Table $\mathrm{V}$ shows that senior registrars seemed to miss significantly more key words than other grades of staff $(47 \cdot 5 \%$ completeness, $\mathrm{p}<0.05$ ).

Comparison of the database with the hospital statistics for completeness and accuracy showed that the hospital figures were more complete but that the database was much more accurate (both $\mathrm{p}<0.001$ ) and 
therefore there was no significant difference between the overall quality of information in each (fig 3 ).

\section{Discussion}

Any information handling system is only as good as the information it contains. The Manchester orthopaedic database offers certain advantages in ease of information gathering and use. Among these are data collection by a mechanism which is already familiar; no need for additional staff, owing to increased secretarial efficiency; use of an English language key word coding system; and collection of data on all episodes of patient care, including outpatient care.

We were able to show that the information contained is highly accurate, much more so than data collected by the hospital. This suggests that users have grasped the principles of the key word system. Of course, even $96 \%$ accuracy leaves some room for improvement.

It is disappointing, however, to find that $38 \%$ of the key words which should have been in the database were missing. This seems simply to be because the doctors concerned forgot to dictate them. We found no evidence that key words were being dictated but subsequently failed to appear in the database or that they were lost once inserted. Lyons and Gumpert found a similar incidence of incomplete records, ${ }^{5}$ and Coleman et al found that $37 \%$ of inpatient information was not recorded. ${ }^{7}$ These authors collected data on only inpatient episodes, and no measure of the accuracy of their information was provided. Our study disclosed that $30 \%$ of our inpatient data was missing; for outpatients, presumably because of doctors' increased pressure of work, completeness of the data was even lower.

The most serious discrepancies were in recording complications, where over half were missing. This has important implications for audit of the unit's activities Scarcely less serious implications attach to underrecording of operations performed and patients admitted, as these will form the basis of hospital financing in the "reformed" health service.

The current key word record is printed in the case notes. It was intended that when a patient was seen again this record should have been scrutinised and, if necessary, corrected. This quality control mechanism was not working properly: only a fifth of required corrections were made at any time, although eventually about half appeared in the course of repeated patient encounters.

The ordinary medical case record is known to be deficient in many respects, ${ }^{89}$ implying that the

TABLE IV-Completeness and accuracy for different key word indications

\begin{tabular}{lccc}
\hline & \multicolumn{2}{c}{ New key } \\
word needed & $\begin{array}{c}\text { Missing key } \\
\text { word }\end{array}$ & $\begin{array}{c}\text { Wrong key } \\
\text { word }\end{array}$ \\
\hline No of key word opportunities & 451 & 121 & 33 \\
No of key words present & 341 & 23 & 9 \\
Completeness (\%) & $75 \cdot 6$ & $19 \cdot 0$ & $27 \cdot 3$ \\
No of key words correct & 332 & 22 & 6 \\
Accuracy (\%) & $97 \cdot 4$ & $95 \cdot 7$ & $66 \cdot 7$ \\
\hline
\end{tabular}

Difference between new key word $v$ missing key word for completeness $\mathrm{p}<0.001$, new key word $v$ wrong key word $\mathrm{p}<0.001$; all other comparison not significant.

TABLE V-Completeness and accuracy by grade of user

\begin{tabular}{lcccc}
\hline & Consultant & Senior registrar & Registrar & Senior house officer \\
\hline No of key word opportunities & 294 & 158 & 123 & 30 \\
No of key words present & 194 & 75 & 84 & 20 \\
Completeness (\%) & $66 \cdot 0$ & $47 \cdot 5$ & $68 \cdot 3$ & $66 \cdot 7$ \\
No of key words correct & 187 & 72 & 82 & 19 \\
Accuracy (\%) & $96 \cdot 4$ & $96 \cdot 0$ & $97 \cdot 6$ & $95 \cdot 0$ \\
\hline
\end{tabular}

$\mathrm{X}^{2}=10 \cdot 1, \mathrm{p}<0 \cdot 05$ for completeness. completeness of the key word record may actually be overestimated. We tried to minimise this by using every available source of information in deriving the ideal key word record, including not only the medical notes but also nursing records, laboratory and radiological reports, and letters.
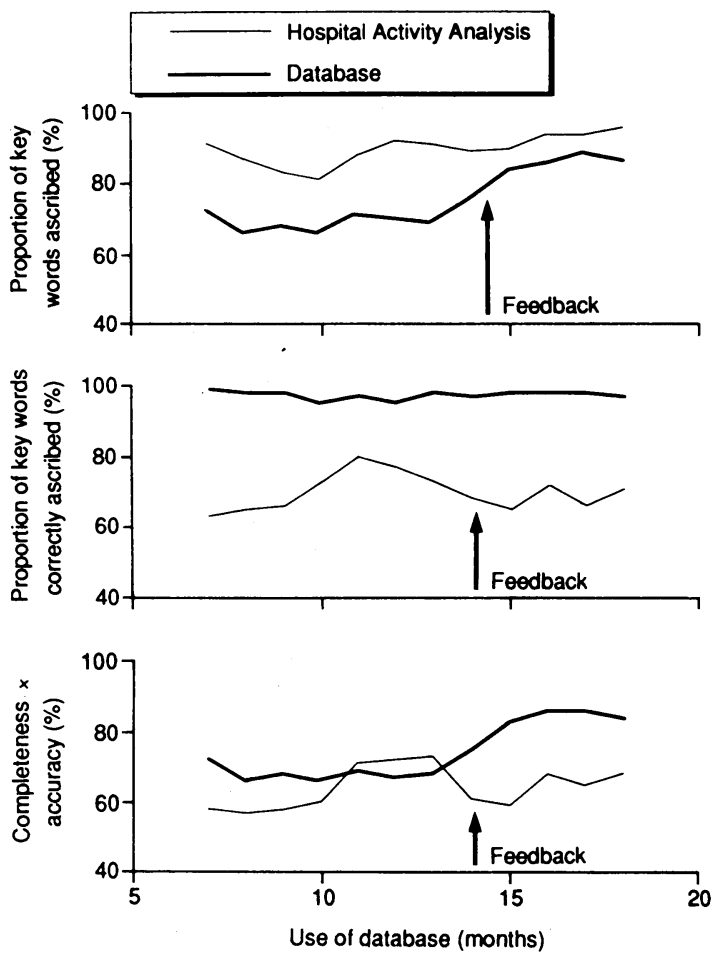

FIG 3-Comparison of data from database and Hospital Activity Analysis for completeness (top), accuracy (middle), and final quality (bottom)

More encouraging was the finding that discussion of key words led to an improvement in completeness of the records. This supports our overall feeling that motivation is the most effective factor in promoting correct use of the database.

Before the introduction of the database the main sources of information for audit and research were, on the one hand, Hospital Activity Analysis and Körner data (which are known to be inaccurate ${ }^{10-12}$ and out of date $^{13}$ ) and on the other, clinical and similar records, such as operating theatre log books, which are not only inaccurate but also extremely time consuming to retrieve information from. Whates et al found the accuracy of Hospital Activity Analysis data to be about $90 \%$ and completeness about $60 \%{ }^{10}$; interestingly, we found about $60 \%$ accuracy and $90 \%$ completeness of Hospital Activity Analysis data. Sunderland found a wide range of inaccuracy in Hospital Activity Analysis reporting of rhesus isoimmunisation, both appreciable underestimates and overestimates, and numerous codings that were totally inexplicable. ${ }^{12} \mathrm{We}$ too found several incomprehensible coding mistakes, such as an arthroscopic meniscectomy coded as "skeletal traction." We are forced to conclude that despite instruction coding clerks sometimes find it impossible to understand medical notes. Furthermore, Hospital Activity Analysis does not give any information on outpatient care. The Manchester orthopaedic database therefore represents a real improvement in clinical information gathering.

We remain convinced that the key word method of data capture is good. This provides a flexible, easily mastered coding system ${ }^{14}$ which, however, is also powerful enough to translate to other widely adopted codes such as the International Classification of Diseases or the Read coding system.

There are clearly aspects of the database that need 
improvement. These lie principally in educating and encouraging users to remember to enter appropriate and accurate key words and to check the completed record at each consultation, especially at the end of a period of care. Discussion of key word use at unit audit meetings and feedback to users have been shown to be of value. Further assessment will be necessary to see whether this effect is maintained.

In addition, and as a direct result of this study, we have started to modify the software to produce discharge summaries in a different way. Instead of consisting of free text dictated by a member of the medical staff it will now be generated automatically from the key word record in the database. The obligation to provide an accurate discharge summary will act as a stimulus to the medical staff to check that the record is complete and accurate. ${ }^{15}$

We thank Professor C S B Galasko, who had the original concept of the design of the Manchester orthopaedic database; Key Computers, which developed it; the consultants, junior medical staff, and secretaries at Ancoats Hospital; and the medical records staff for their help with collecting data for the study.
1 Ellis BW, Michie HR, Esufali ST, Pyper RJD, Dudley HAF. Development of a microcomputer-based system for surgical audit and patient administration: a review. F $R$ Soc Med 1987;80:157-61.

2 Dunn DC. Audit of a surgical firm by microcomputer: five years' experience. BMF 1988;296:687-91.

3 Brand DA, Krag MH, Hausman MR, Trainor KF, Akelman E, Rudicel SA et al. A patient registry for orthopaedic surgery. Clin Orhop 1990;252:262-9.

4 Kuslich SD, Peck CM. The human element in the design of computer assisted orthopaedic inpatient medical records systems. Orhop Clin North Am orthopaedic inparie

5 Lyons C, Gumpert R. Medical audit data: counting is not enough. $B M \mathcal{J}$

6 Marsh DR, Galasko CSB. The Manchester orthopaedic database. $\mathcal{F}$ Bone foin Surg [Br] 1990;72B:933.

7 Coleman NP, Warren PJ, Greenough CG. Accuracy and completeness of orthopaedic computer audit. $\mathcal{I} R$ Coll Surg $E$ dinb (in press).

8 Swansea Physicians' Audit Group. Audit of the quality of medical records in a district general medicine unit. F $R$ Coll Physicians Lond 1983;17:208-12.

9 Heath DA. Random review of hospital patient records. BMF 1990;300:651-2.

10 Whates PD, Birgzalis AR, Irvine M. Accuracy of hospital activity analysis codes. $B M \mathcal{F}$ 1982;284:1857-8.

11 Rees JL. Accuracy of hospital activity analysis data in estimating the incidence of proximal femoral fractures. $B M \mathcal{F} 1982 ; 284: 1856-7$.

12 Sunderland R. Inaccurate coding corrupts medical information. Arch Dis Child 1985;60:593-4.

13 Yates JM, Davidge MG. Can you measure performance? BMF 1984;288: $1935-6$

14 Kurtz D, Morrish KA. A microcomputer approach to storage of qualitative patient data using key words. Comput Biomed Res 1983;16:209-17.

15 Smith RP, Holzman GB. Application of a computer database system to generation of hospital discharge summaries. Obstet Gynecol 1989;73:803-7.

(Accepted 8 October 1991)

\title{
International Quality Assurance
}

\section{Quality assurance in Finland}

\author{
Hannu Vuori
}

\section{A failed head start}

Finland once tried to make a head start with quality assurance. While most of Europe was still slumbering like Sleeping Beauty, it flirted with quality assurance in the early 1970s. In 1971 the Finnish Hospital League urged all hospitals to start medical audit. Today, 20 years later, not a single hospital has heeded the advice. How did quality assurance start and why did it fail?

As the recommendation for starting medical audit resulted from my doctoral dissertation, ${ }^{1}$ I shall venture to look at its development from a personal point of view. It all started from an academic interest. When I came across quality assurance, at the University of California, Berkeley, during the mid-1960s I believed that the ideas must be applicable in Finland. Back in Finland I launched a study with two aims: to assess whether medical audit is possible in Finnish hospitals and whether assessors with different levels of medical knowledge (physicians, nurses, and lay people) use objective criteria to screen medical records for poor quality in the same way. The experience of the United States shows that quality assurance is a hot potato. I therefore carefully tried to avoid stepping on people's toes. My first choice was to focus on the methods of quality assurance, not on the actual quality of care delivered. My second choice concerned my coworkers. Working in a department of social medicine, I was an outsider; I knew that I could not gain the confidence of my clinical colleagues without involving them. I asked them to develop the criteria of good care. I also tried to consult the respective professors at every major step of the study.

The study reached its goals. But it also showed that my fears had been warranted. Though the younger clinicians were sympathetic and cooperative, the older clinicians, particularly the professors, tried to sink the study - and with it the spectre of quality assurance.

What went wrong? The study provided some probably universal lessons. Firstly, that because quality assurance is a practical venture to solve problems in patient care, an academic interest is not the best starting point. It does not convince the clinicians that quality assurance is useful; at best, they find it a nuisance, at worst a threat. Secondly, that outsiders, particularly those from other disciplines, are unwelcome intruders. It is not enough to inform and involve clinicians; they should do most of the work. The greatest mistake was perhaps the recommendation put forward by the Hospital League. As the league represents the hospital owners, the clinicians interpreted the move as a veiled hint that "big brother" would like to take control.

\section{Long detour}

Quality assurance only takes off if you can show a need for it. The Finnish clinicians were content with the situation. If they had heard of quality assurance, they found it relevant only for big countries, such as the United States, with many educational institutions and a great variety of treatment facilities. They accepted that under those circumstances the quality of care can vary and that systematic attempts to measure and improve it may be needed. Why should Finland need quality assurance? they asked. The country is small and has only a handful of medical schools that follow roughly the same curriculum. The care in hospitals and primary health care centres is consistently of high quality. The current societal and professional control mechanisms suffice to take care of the rare rotten apples.

Applied health research shattered this complacent view. In 1972 the Finnish Medical Research Council approved the country's first health research policy. Among the new priorities were epidemiology and health services research. The new generation of epidemiologists and health services researchers, trained with the support of the research council, became interested in small area variation. In study after study they showed variation in infant mortality among 